\title{
Thoracodorsal artery flaps for breast reconstruction-the variants and its approach
}

\author{
Jørn Bo Thomsen ${ }^{1}$, Mikkel Børsen Rindom ${ }^{2,3}$, Alberto Rancati ${ }^{4}$, Claudio Angrigiani ${ }^{4}$ \\ ${ }^{1}$ Research Unit for Plastic Surgery, Odense University Hospital, University of Southern Denmark, Odense; \\ ${ }^{2}$ Department of Plastic Surgery, Odense University Hospital, Odense; ${ }^{3}$ Department of Plastic Surgery, Lillebaelt Hospital, Vejle, Denmark; \\ ${ }^{4}$ Instituto Oncologico Henry Moore (IOHM), Universidad de Buenos Aires, Buenos Aires, Argentina
}

\begin{abstract}
Thoracodorsal artery (TDA) flaps, ranging from the vascular-pedicled thoracodorsal artery perforator (TDAP) flap, the propeller TDAP flap, and the muscle-sparing latissimus dorsi (MSLD) flap to the conventional latissimus dorsi (LD) flap and the extended LD flap, can all be used for breast reconstruction. The aim of this paper and review is to share our experiences and recommendations for procedure selection when applying TDA-based flaps for breast reconstruction. We describe the different surgical techniques and our thoughts and experience regarding indications and selection between these procedures for individual patients who opt for breast reconstruction. We have performed 574 TDA flaps in 491 patients: 60 extended LD flaps, 122 conventional LD flaps, two MSLD flaps, 233 propeller TDAP flaps, 122 TDAP flaps, and 35 free contralateral TDAP flaps for stacked TDAP breast reconstruction. All the TDA flaps are important flaps for reconstruction of the breast. The LD flap is still an option, although we prefer flaps without muscle when possible. The vascular-pedicled TDAP flap is an option for experienced surgeons, and the propeller TDAP flap can be used in most reconstructive cases of the breast, although a secondary procedure is often necessary for correction of the pedicle bulk. The extended LD flap is an option for women with a substantial body mass index, although it is associated with the highest morbidity of all the TDA flaps. The MSLD flap can be used if the perforators are small or if dissection of the perforators is assessed to be hazardous.
\end{abstract}

Keywords Breast / Surgical flaps / Education, medical
Correspondence: Jørn Bo Thomsen Research Unit for Plastic Surgery, Odense University Hospital, University of Southern Denmark, J.B. Winsløws Vej 4, Odense 5000, Denmark Tel: $+45-29270029$

Fax: $+45-65413038$

E-mail: Joern.Bo.Thomsen@rsyd.dk

\section{INTRODUCTION}

The latissimus dorsi (LD) muscle flap was first described by Tansini in 1906 [1]. According to Maxwell [2], it was used in Europe between 1910 and 1920. Use of the flap, however, largely remained dormant in the literature until it was described for breast reconstruction in combination with an implant by Schneider in 1977 [3]. The original myocutaneous flap design was later extended for complete autologous reconstruction by including larger amounts of subcutaneous fat as described by Hokin [4], resulting in the so-called extended LD (ELD) flap.

An increased focus on donor site morbidity and preservation of function, as exemplified by the evolution of Hartrampf's transverse rectus abdominis myocutaneous (TRAM) flap to Holmström's free abdominoplasty flap and later Allen's fasciocutaneous deep inferior epigastric artery perforator (DIEP) flap, 
has also influenced views on the classical LD flap design [5-7]. Tobin initiated this era in the 1980s, when tailoring the LD flap and other muscle flaps using only segments of the muscle, leaving functional muscle behind, and Maruyama described that using the horizontal section of the muscle enabled a fasciocutaneous extension to diminish flap bulkiness [8,9].

Inspired by the above innovations, the "latissimus dorsi flap without muscle" was described in 1995 [7]. This was the first thoracodorsal artery perforator (TDAP) flap. Since then, several different variations of the original TDAP technique have been described. Along with this development, different versions of a muscle-sparing LD (MSLD) flap and different designs of a propeller TDAP flap have been presented [7-13].

The transition from the TRAM flap to the DIEP flap, as well as the transition from the LD flap to the TDAP flap, is exemplified in publications by Nahabedian et al. [14] and Hamdi et al. [9] describing the selection criteria of the different procedures.

The TDAP flap and its variations can all be considered modifications or improvements of the original LD myocutaneous flap $[10,15]$. This development can be attributed to the perforator flap techniques, where some of the cutaneous branches of the thoracodorsal artery (TDA) are identified and dissected from the muscle, resulting in a cutaneous paddle [7]. The advantages and disadvantages of this technique have previously been described in the literature $[9,12,16]$.

The common denominator for these flaps is that they are all variations of pedicled TDA flaps, which can be used for breast reconstruction. The flaps are not identical, but they can be considered a surgical "finesse" of the same procedure. The indications for use of the different TDA flaps are thus not the same. Surgeons tend to focus on patient selection for specific procedures. However, the different types of TDA flaps should, per- haps, be selected based on the patient instead — that is, a procedure should be selected for a specific patient.

The aim of this paper and review is to present our clinical experience and recommendations for procedure selection when applying TDA-based flaps for breast reconstruction.

\section{OUREXPERIENCE USING TDA FLAPS}

We present a review of the literature combined with a retrospective review of data on women undergoing breast reconstruction with different TDA flaps performed in Denmark, Norway, and Argentina over a 20-year period between 1998 and 2018. The data from Argentina were approved by the Ethics Committee of Instituto Oncológico Henry Moore (IRB No. HM-12008). The Scandinavian data are a compilation and summary of previously published data and derive from a study approved by the Danish Committee on Health Research Ethics (S-20120207) [10,15, 17]. The data and images presented in this paper are published with consent of the involved patients. Based on our experience, we describe our criteria for procedure selection and indications.

\section{Surgical technique \\ The classic TDAP flap}

The cutaneous paddle of the TDAP flap is dissected entirely of the underlying muscle, followed by the dissection of one or more perforators through the muscle to the thoracodorsal bundle [7]. The classical TDAP flap is illustrated in Fig. 1, showing the vascular-pedicled flap. The classical TDAP flap is associated with very few complications/comorbidities, when raised correctly. However, dissection of the flap is challenging, and flap loss is a possible major complication. If the length of the vascular pedicle is sufficient, there is no need to divide the thoracodorsal vessels

\section{Fig. 1. The classical TDAP flap}

Illustration of the classical thoracodorsal artery perforator (TDAP) flap raised on the vascular pedicle.
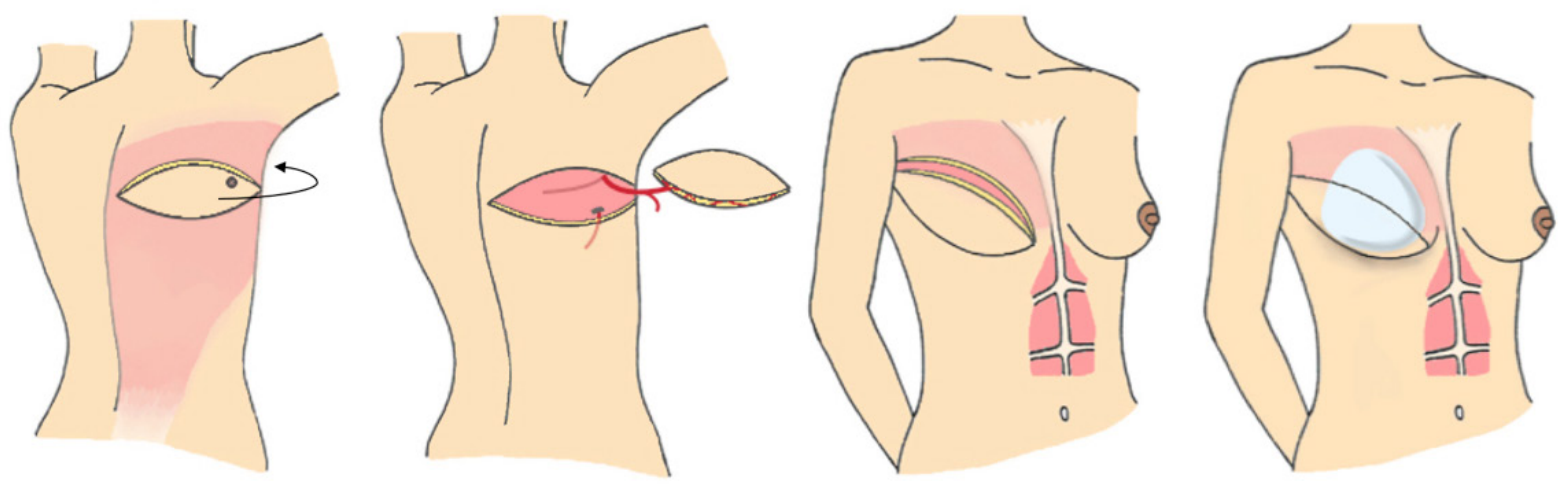
for additional lengthening. However, division of the vascular bundle is often needed if the flap reaches the medial part of the breast. This should be done without causing damage to the thoracodorsal nerve.

\section{The propeller TDAP flap}

The propeller TDAP flap is a simplified TDAP procedure where dissection around the perforator is more limited [10$13,18]$. The skin island is still dissected of the underlying muscle, but only until the flap can be turned as a propeller. The location where the perforator pierces the muscle fascia is the turning point (Figs. 2, 3). The propeller TDAP flap is illustrated in Fig. 4 , showing the flap rotated around the perforator to the anterior part of the thorax. The main complications are venous conges-

\section{Fig. 2. Raising the propeller TDAP flap}

Delayed breast reconstruction with a propeller thoracodorsal artery perforator (TDAP) flap. (A) Drawings, (B) identification of the perforator, (C) raising the flap, and (D) closure of the donor site.
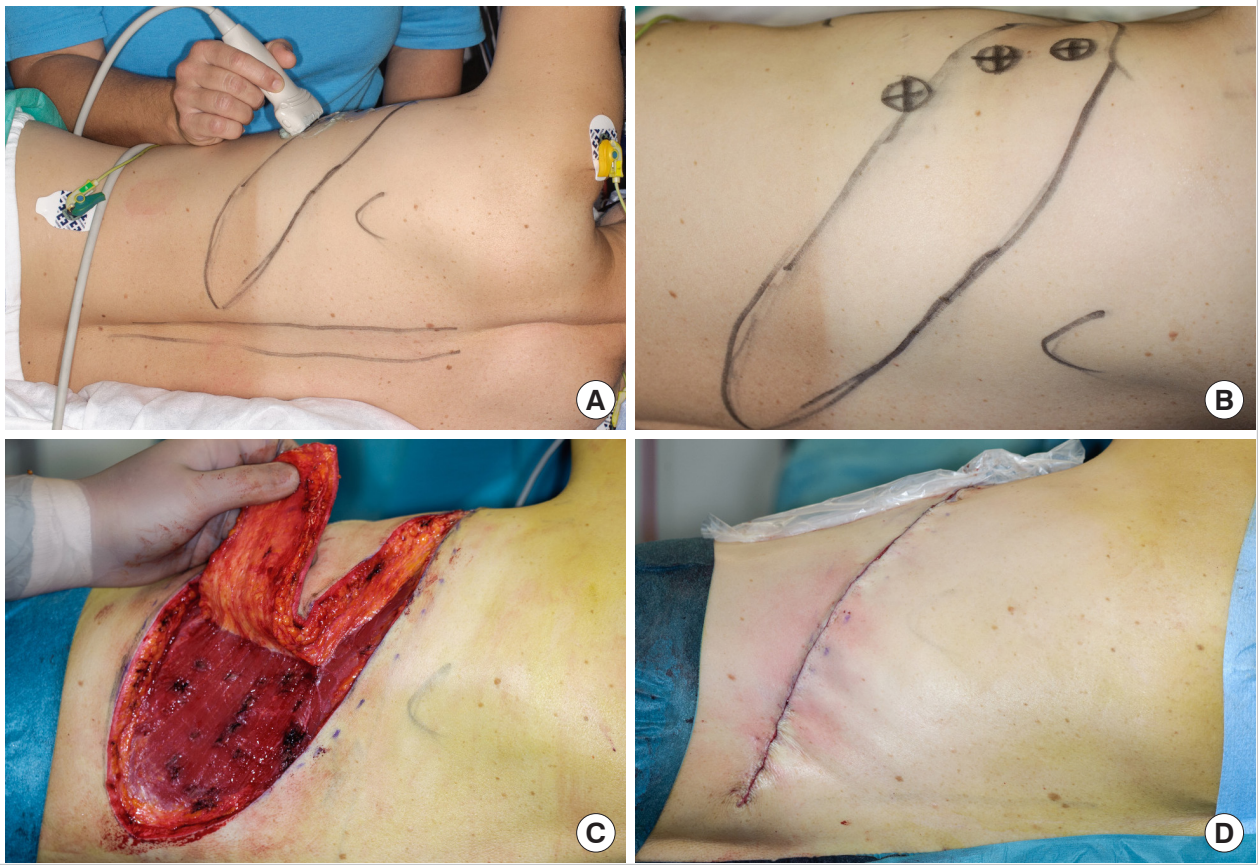

Fig. 3. Reconstruction with implant and propeller TDAP flap

(A) The propeller thoracodorsal artery perforator (TDAP) flap raised and ready for reconstruction. (B) The recipient site/flap raised including skin and pectoralis major muscle. (C) The TDAP flap placed in the inframammary crease. (D) The completed reconstruction using a TDAP flap and an implant.
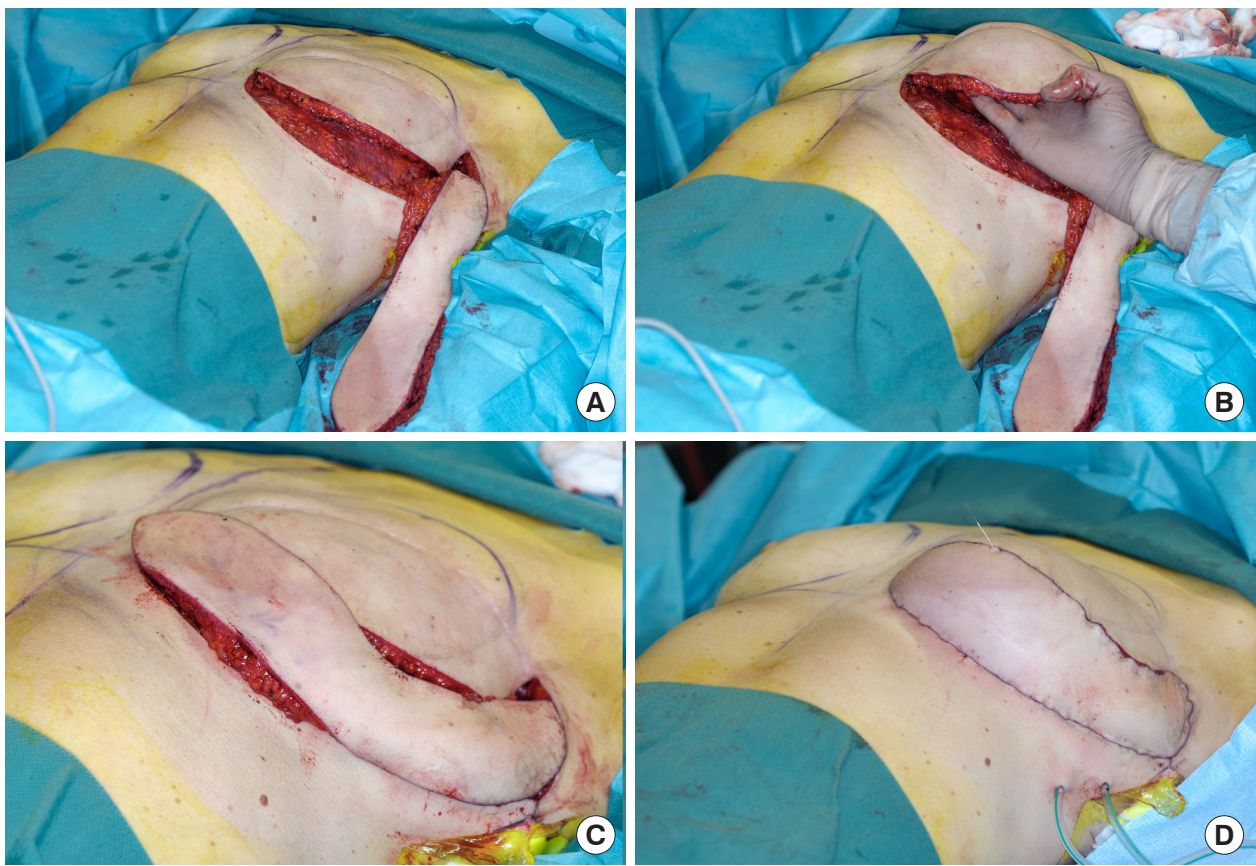


\section{Fig. 4. The propeller TDAP flap}

Illustration of the propeller thoracodorsal artery perforator (TDAP) flap, rotating the flap around the perforator.
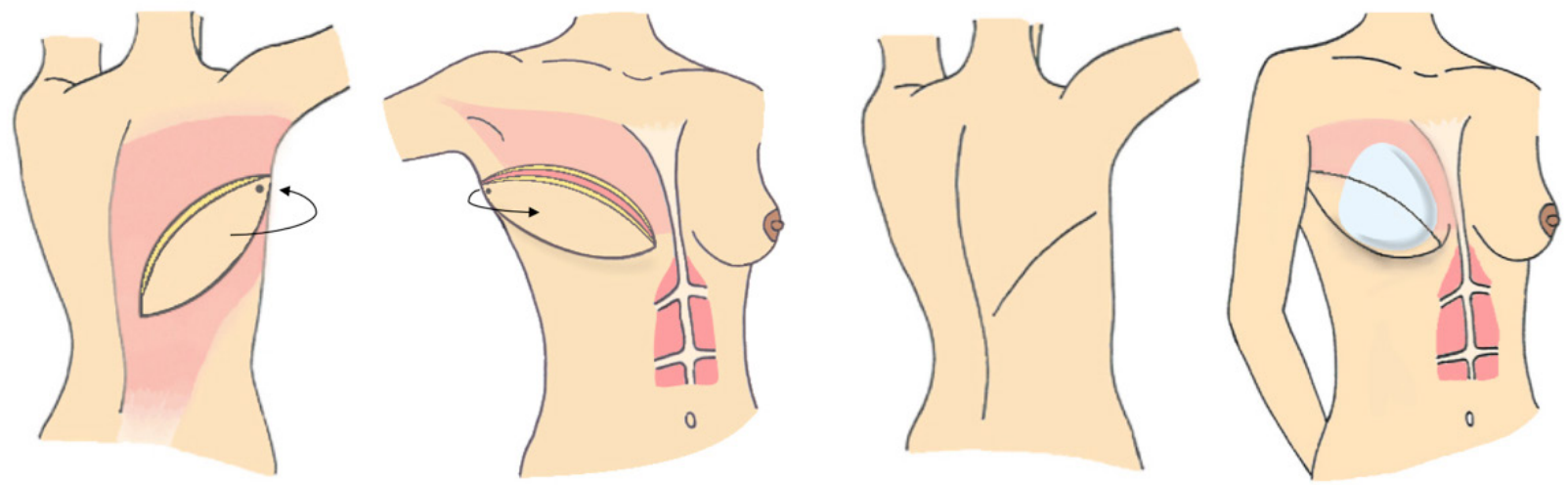

\section{Fig. 5. Propeller TDAP reconstruction combined with fat grafting}

Breast reconstruction with a propeller thoracodorsal artery perforator (TDAP) flap in combination with fat grafting. (A, B) Prior to mastectomy of left breast and prior to mastopexy of right breast. (C, D) After left-sided mastectomy and reconstruction with propeller TDAP flap and fat grafting and after rightsided mastopexy.
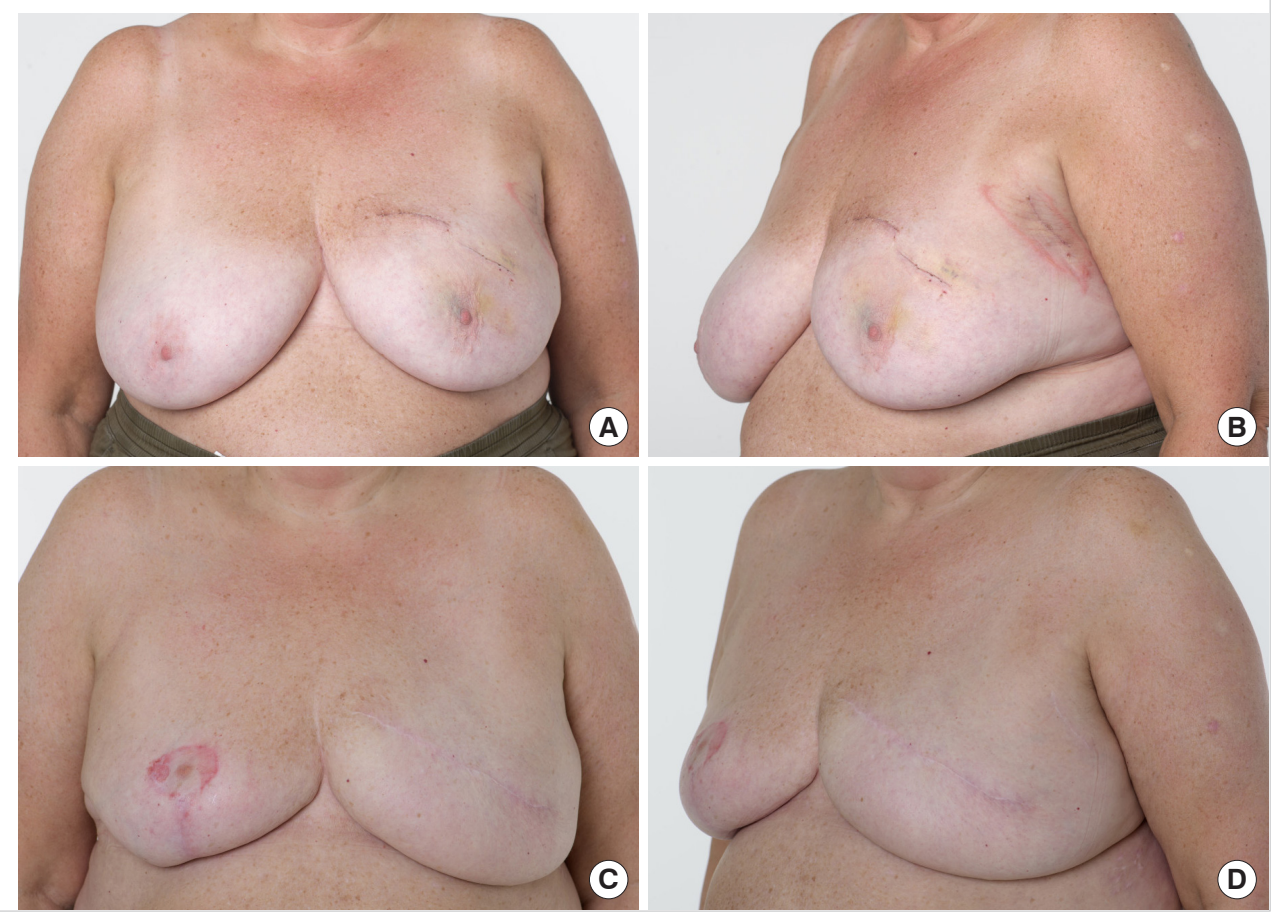

tion in the third angiosome when harvesting a large flap, and the risk of general venous congestion due to compromised venous outflow at the turning point of the perforator. The flap can be augmented in several ways, including by an implant (Fig. 3) or by fat grafting (Fig. 5). The propeller TDAP flap can be either perforator-pedicled or subcutaneous-pedicled [19].

\section{The MSLD flap}

The MSLD flap is raised with the vertical and anterior part of the LD muscle, containing the descending branch of the TDA, included in the pedicle. The MSLD flap is illustrated in Fig. 6, showing the flap with its pedicle in the anterior vertical part of the LD muscle, including the vertical branch of the thoracodorsal vessels and adjacent perforators. The flap reach is almost the same as that of the classical TDAP flap. Distal venous congestion in the third angiosome can pose a problem. In theory, using the flap may lead to LD muscle function morbidity. However, the literature related to this is scarce. The skin paddle can be placed in various locations on the back, as long as its basis is placed on top of a sizable portion of the muscle pedicle, including one or more perforators. The MSLD flap can be viewed as a muscle-pedicled propeller flap [19]. 


\section{Fig. 6. MSLD breast reconstruction with implant}

Illustration of the muscle-sparing latissimus dorsi (MSLD) flap transposed on the muscle pedicle, including the descending branch of the thoracodorsal vessels.
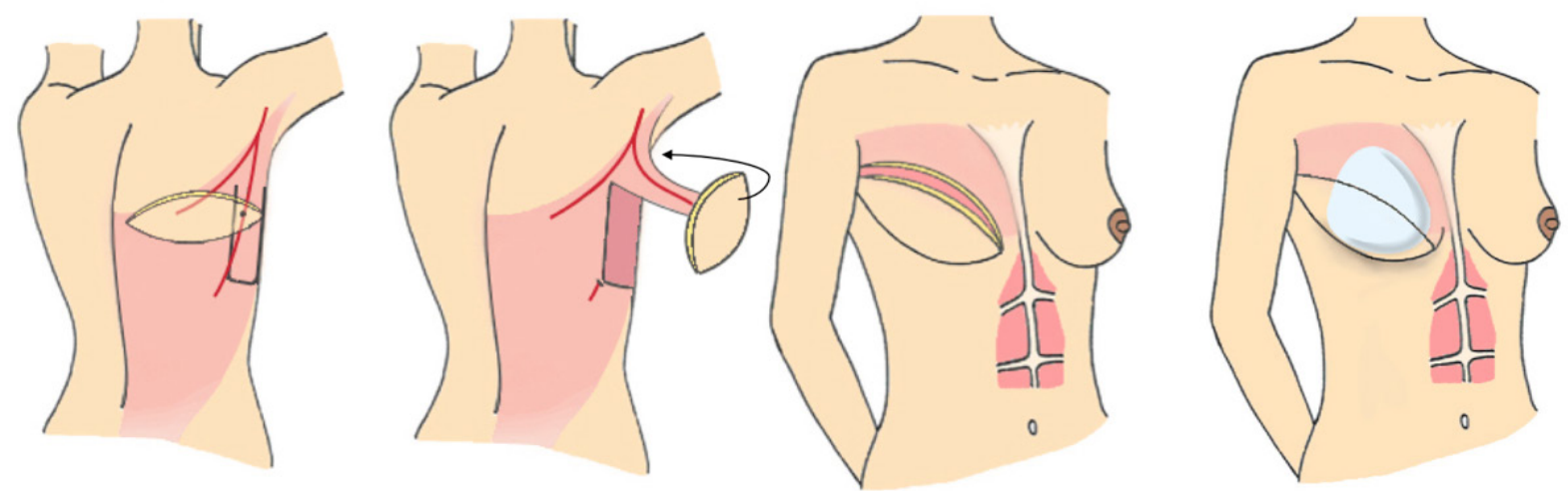

\section{Fig. 7. LD breast reconstruction with implant}

Illustration of the latissimus dorsi (LD) flap showing the utilization of the whole LD muscle as the pedicle and for reconstruction.
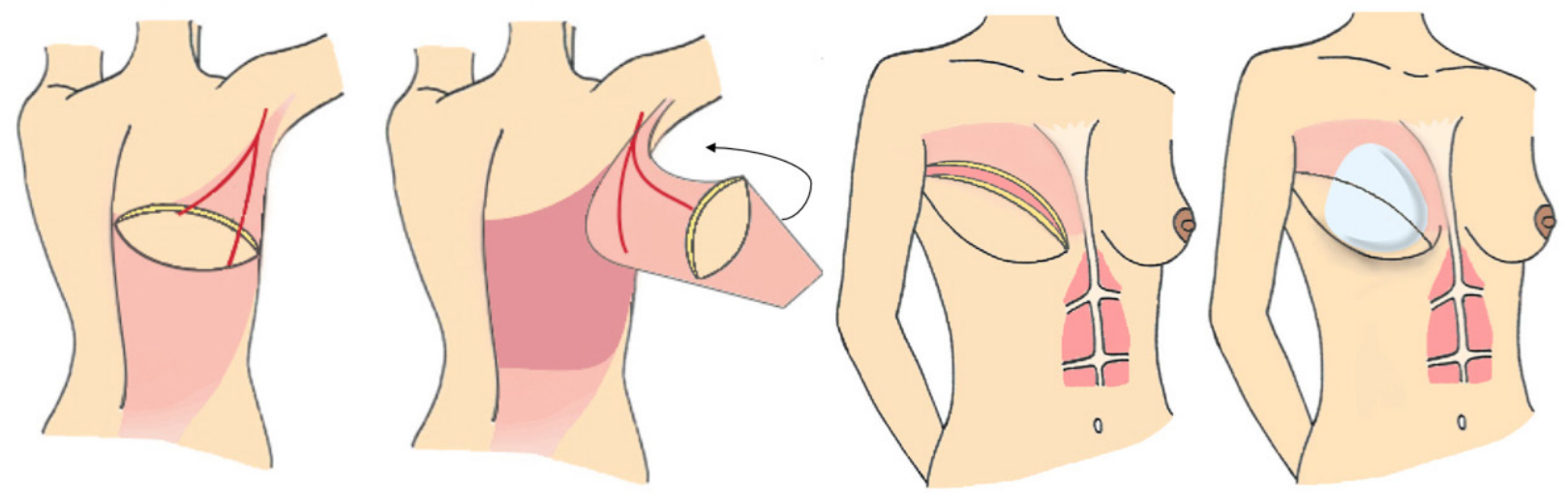

\section{The conventional LD flap}

The conventional LD flap is raised as a skin paddle carried on the entire underlying LD muscle. The muscle is released from its insertions on the trunk and often, to increase its reach, from its insertion on the humerus. The LD flap is illustrated in Fig. 7, showing the flap with the LD muscle as its pedicle. This flap is associated with shoulder and arm morbidity, as well as seroma formation at the donor site. The thoracodorsal nerve can either be left intact or divided. If it is left intact, there is a potential risk of breast animation deformity, and if it is divided, the muscle will undergo atrophy over time.

\section{The ELD flap}

The ELD flap and the conventional LD flap are raised in the same manner, but the extended version contains a maximum amount of subcutaneous soft tissue on top of the LD muscle
[20-22]. The ELD flap is illustrated in Fig. 8, showing the flap with the LD muscle, including the subcutaneous fat, as its pedicle. This flap is associated with shoulder and arm morbidity, as well as seroma formation at the donor site. The contour of the back/donor site is affected by the missing soft tissue. When using the ELD flap, there seems to be an increased risk of wound dehiscence and prolonged healing at the donor site. The markings for the maximal-sized skin island are based on experience and pinch tests. The skin island can be placed in different positions according to preference. However, if the paddle is located on the cranial part of the muscle, the attachment to the humerus must be divided for better reach. As with the other TDA flaps, the ELD flap can be combined with fat grafting (Fig. 9). The fascia of Scarpa should be preserved for closure of the donor site if possible. 


\section{Fig. 8. ELD flap breast reconstruction}

Illustration of the extended latissimus dorsi (ELD) flap, where the maximum amount of fat is harvested with the latissimus dorsi flap for total reconstruction.
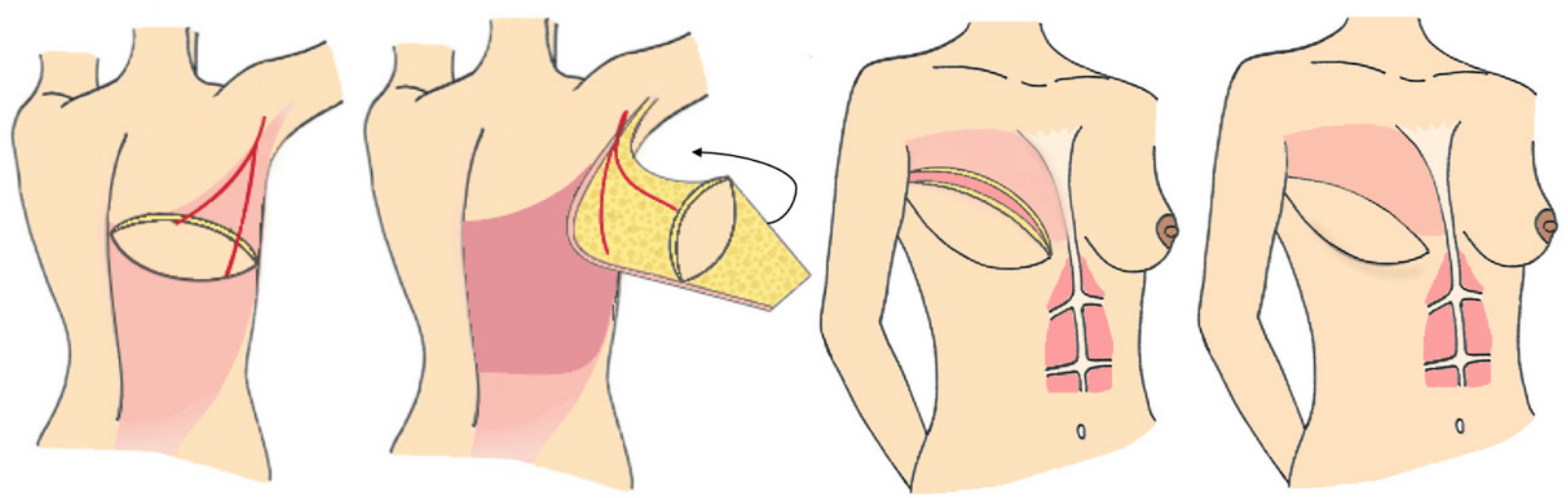

\section{Fig. 9. ELD flap reconstruction with fat grafting}

Picture showing the drawings for an extended latissimus dorsi (ELD) flap harvested with fat, the combination with fat transplantation for added volume, and the reconstructed breast. (A) The markings of the ELD flap. (B) The ELD flap raised with maximum amount of tissue. (C) Fat grafting of the ELD flap. (D) The breast reconstructed with an ELD flap combined with peroperative fat grafting.
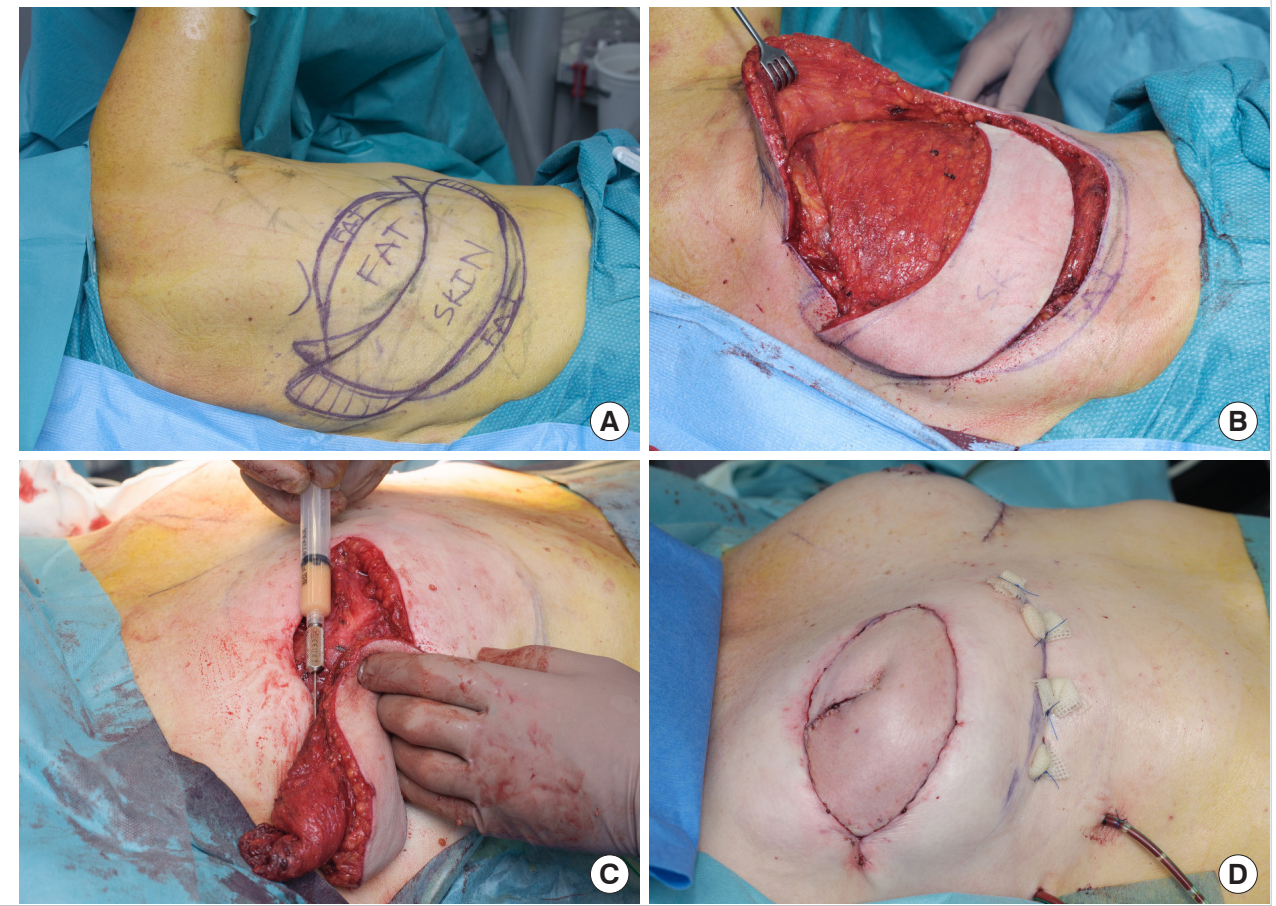

\section{Indications and procedure selection}

\section{The TDAP, propeller TDAP, and MSLD flaps}

The muscle-sparing versions of the TDA flaps (TDAP flap, propeller TDAP flap, and MSLD flap) are generally indicated in cases when preservation of LD muscle function is desired. These flaps can be applied in women who require moderate amounts of soft tissue transfer and when one or more suitable perforators can be identified. There are, however, different advantages and disadvantages to be considered for each design.

The classic TDAP flap should only be applied when a sizable perforator of the TDA is present and when the surgeon is sure that the perforator can safely be dissected through the muscle to the feeding vessels without compromising the blood flow.

The propeller TDAP flap can be applied as an alternative in most cases where perforator(s) can be identified, regardless of the size. Identification can be done safely either freestyle or preferably targeted by color Doppler ultrasonography (CDU) $[10$, $11,13,16,18]$.

Both the conventional and propeller TDAP flaps are normally combined with an implant for sufficient reconstruction of the 
breast contour, but in select cases these flaps can be used for a total autologous breast reconstruction [16], in combination with a free TDAP flap as stacked flaps or together with fat grafting (Fig. 5).

The MSLD flap is indicated in more complex cases, with limited radiation damage and acceptable skin flap thickness in the recipient area. The flap can be used as an alternative to the propeller TDAP flap to ensure sufficient perfusion of the flap [12]. The MSLD flap is chosen instead of in the other muscle-sparing flaps in the following cases: (1) When the perforator(s) are small; (2) When the surgeon is uncertain whether the perforators can be identified and dissected sufficiently; (3) When the design of the skin paddle cannot be placed on the area of a sizable perforator of the descending branch of the TD vessels; (4) When previous surgery has left a scar that involves the vascularization of the first perforator; (5) When an alternative perforator cannot be identified in a relevant location by CDU; or (6) When the skin island is harvested for nipple-areolar complex resurfacing only, and the pedicle must be placed distal to the area where the first perforator pierces the fascia.

\section{The LD flap and ELD flap}

The full LD flaps are indicated in cases with severe radiation damage and/or very thin skin in the recipient area. In these cases, the additional vascularized soft tissue provided by the muscle is required for sufficient coverage of the implant.

Some patients opt for $\mathrm{LD}$ reconstruction rather than a perforator flap. In women who actively engage in sports, we aim to leave the muscle function intact and advise the use of perforatorbased flaps due to the risk of shoulder and arm-related morbidity following LD flap harvest $[15,20]$.

The ELD flap can be indicated/selected in women with a high body mass index (BMI) and localized fat deposits in the back [20] where other reconstructive options are less favorable [21]. Harvest of the flap does, however, come with a risk of donor site seroma.

\section{Augmentation of TDA flaps}

Any of the TDA flaps can be augmented by adding an implant, by adding an additional perforator flap, pedicled or free, as stacked flaps, and/or by combining the procedure with fat grafting.

\section{OUREXPERIENCE USING TDA FLAP BREAST RECONSTRUCTIONS}

A retrospective review of our own experience using TDA flaps included 491 cases of breast reconstruction in women with TDA flaps conducted over a 20 -year period. In total, we performed
Table 1. Our experience using TDA flap for breast reconstruction

\begin{tabular}{lccr}
\hline Variable & $\begin{array}{c}\text { No. of } \\
\text { patients }\end{array}$ & $\begin{array}{c}\text { No. of } \\
\text { bilateral flaps }\end{array}$ & $\begin{array}{c}\text { No. of } \\
\text { flaps }\end{array}$ \\
\hline Extended LD & 42 & 18 & 60 \\
Conventional LD & 103 & 19 & 122 \\
MSLD & 2 & 0 & 2 \\
TDAP propeller & 205 & 28 & 233 \\
TDAP & 104 & 18 & 122 \\
Free TDAP & 35 & 0 & 35 \\
Total & 491 & 83 & 574 \\
\hline
\end{tabular}

TDA, thoracodorsal artery; LD, latissimus dorsi; MSLD, muscle-sparing LD; TDAP, thoracodorsal artery perforator.

574 TDA flaps in 491 patients ( 408 unilateral cases and 83 bilateral cases). The procedures included 60 ELD flaps (10\%), 122 conventional LD flaps (21\%), two MSLD flaps (0\%), 233 propeller TDAP flaps (41\%), 122 TDAP flaps (21\%), and 35 free contralateral TDAP flaps for stacked TDAP breast reconstruction (6\%) (Table 1). We achieved the planned breast reconstruction in 459 of 491 cases (93\%). We have not included details about flap size, aesthetic outcomes, or complications of the individual flap types in this review, as the majority of our data have been published previously, for the classic TDAP flap $[7,16$, 23], the propeller TDAP flap [10-13,18-20,23-25], LD flaps $[7,19,20,24,26]$, and the stacked TDAP flap [27].

\section{DISCUSSION}

Application of the muscle-sparing versions of the TDA flaps for breast reconstruction is increasing worldwide with a rising number of publications reporting the utilization of these flaps for both immediate and delayed breast reconstruction $[8,9,11,16$, 18,28-30]. In our clinics, the ratio of perforator-based TDA flap breast reconstructions to myocutaneous TDA flap-reconstructions has increased over time.

The difficulty of TDA flap application ranges from the relatively easy harvest of the classical LD flap to the somewhat demanding TDAP flap. We increasingly try to avoid inclusion of muscle in our flaps. This naturally comes with increased experience in perforator-based flap surgery in general and TDAP flap surgery in particular.

We still apply all the different types of TDA flaps for breast reconstruction. The choice of a flap depends primarily on the needs and characteristics of a specific patient. Comorbidities are important; if the patient has a significant comorbidity (e.g., hypertension), is an active smoker, or recently stopped smoking, we always recommend the safer $\mathrm{LD}$ flap $[11,12,31]$.

The recipient site also affects the choice of procedure. If mastectomy has left the patient with very thin skin flaps or radiation 
therapy has caused significant sequelae to the skin and subcutaneous tissue, we also recommend use of the LD flap to provide sufficient well-perfused soft tissue for the reconstruction.

The donor site can be affected by previous surgery leaving a scar close to the perforators that makes the blood supply of perforator-based flaps questionable. Furthermore, there may be anatomical variations, revealing multiple small perforators or a dominant perforator deriving from vessels other than the descending branch of the TDA $[29,30]$. If a sizable perforator cannot be identified, we aim for muscle-based reconstruction instead of a perforator-based procedure.

The vascularity of the donor tissue is the most important factor when selecting the type of TDA for reconstruction. The TDA procedure selection can be guided by CDU, which enables the surgeon to perform the selected reconstruction as a targeted procedure by identifying all relevant perforators. This can be performed and used in the outpatient clinic prior to surgery to guide the patient to the safest TDA flap option with the least possible donor site morbidity [32-34]. During surgery, CDU can be used to locate the perforators and to obtain an estimate of the perforator size and topography, including anatomical variations $[11,32]$. Some patients do not have a dominant perforator with a classic location feeding the descending branch of the thoracodorsal vessels. In these cases, the dominant perforator may derive from the horizontal branch or even the subscapular vessels $[11,32]$. In cases where there is a scar at the donor site, other perforators may have become dominant because of redirected blood flow.

The TDA procedures and selection between these procedures can be performed without CDU. However, the surgeon should be prepared to change the type of procedure on the fly $[16,18$, $23]$. As always the surgeon should know and rely on common anatomy $[7,16,18]$.

The LD flap is a very reliable technique and cases of insufficient perfusion of the skin island are rare. In these cases, the underlying muscle will support and cover the underlying implant. On the contrary, poorly perfused TDAP flaps do pose a problem, as the underlying implant can be exposed unless the implant is covered by an acellular dermal matrix, which may cover the implant until salvage surgery [12].

The classical TDAP flap, based on a true perforator pedicle, is also a reliable and safe flap when performed by experienced surgeons $[7,9,16,26]$. Since its initial description, several publications have reaffirmed the concept $[7,9,10,26]$. The perforator flap technique allows the surgeon to completely discard the muscle, solving the problem of bulkiness [7]. Whilst on the learning curve for TDAP dissection, it is advisable to identify the perforator, either by careful dissection or by application of targeted CDU. In that way, conversion to the LD flap is still possible $[7,10-13,16,18]$. The design of the TDAP skin paddle is optional and the flap can be placed either horizontally or obliquely on the back. The donor site scar is more difficult to hide with the oblique design, but the flap needs to be rotated only $130^{\circ}$ compared to $180^{\circ}$ with the horizontal design. This may influence venous circulation and prevent congestion if the perforator is not dissected sufficiently $[13,18,24]$.

During surgery, reassuring continuous bleeding from the flap edges can be observed whilst raising the flap in a distal-to-proximal direction. This gives a clear visual indication that a perforator is located in the proximal end of the flap $[7,16,18]$. After visualization of the proximal perforator(s), a decision must be made between the TDAP flap, propeller TDAP flap, and MSLD flap. The choice between the TDAP and MSLD flap, as described by Hamdi and colleagues, can be related to perforator size and the surgeon's experience [8]. When the perforator is sizable, it is possible to dissect the vessel(s) through the muscle to the TDA as a vascular pedicled TDAP. The flap can then be tunneled through the muscle with minimal damage to the muscle $[7,9,16]$. The descending branch of the thoracodorsal vessels can be divided distally to increase the reach of the flap, but in most cases this is not necessary, as the perforator pedicle itself gives an additional pedicle length of $3-4 \mathrm{~cm}[13,16]$.

If there is doubt whether the perforator can be dissected through the muscle, the propeller TDAP flap is an option, which can be transposed on 1-3 perforators, if they are in close proximity with each other. When choosing to perform the propeller TDAP flap, the surgeon should be prepared to dissect the perforators though the muscle. This can be necessary if the location of the perforator(s) is too far from the anterior edge of the LD muscle to ensure adequate rotation of the flap, as well as in cases where two perforators are included in the flap [10]. In the latter cases, the most distal perforator sometimes needs to be dissected towards the most proximal perforator to allow for rotation of the flap (Fig. 10).

If the perforators are very small and difficult to identify, one can opt for a MSLD flap to ensure perfusion of the flap [9]. Muscle-sparing techniques aim to minimize the risk of perforator damage or to incorporate more perforators under the rationale of obtaining better perfusion. However, if a sizable perforator is identified, there is no need for the muscle-sparing approach. If the complete pedicle is mobilized as part of MSLD surgery, a conventional LD flap is probably a better option, as the muscle is often left without function due to nerve damage. By performing a perforator-based TDA flap, the muscle function is better preserved $[7,10,11,16,18]$.

TDAP flaps can be observed clinically with regard to arterial 


\section{Fig. 10. Dissection of the thoracodorsal artery perforator}

Picture showing a distal perforator dissected towards a proximal perforator to allow for rotation of the flap to the anterior part of the thorax. (A) The distal perforator dissected for better blood supply and rotation of the flap. (B) The intact motor nerve branch carefully dissected of the perforator.

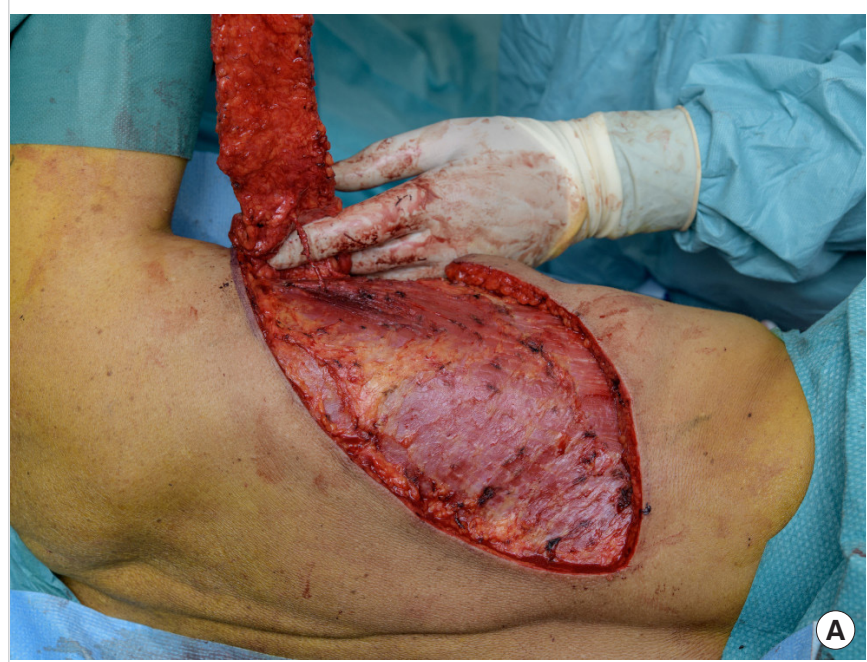

inflow and venous outflow simply by visual assessment of the color, capillary response, and signs of congestion. However, the border between the second and third angiosome can be difficult to assess. Distal flap loss does sometimes occur despite apparent good blood filling based on a clinical assessment [11]. Distal tissue damage in a propeller TDAP is a problem, if it cannot be solved by flap revision. In recent years, it has become possible to prevent these problems by assessing flap perfusion by use of indocyanine green [35]. This assessment often reduces the length of the flap by a couple of centimeters and may save the patient from re-operations $[10]$.

Medial breast defects must be carefully evaluated. These can either be reached and covered by an obliquely designed propeller TDAP flap or a TDAP flap with dissection of the vascular pedicle to the origin of the thoracodorsal vessels to gain the maximum possible pedicle length $[10,16]$. The $\mathrm{LD}$ flap is also a safe and reliable solution for these defects.

In bilateral cases, the risk of raising the flap and the possible morbidity associated with the chosen flap is inherently higher. We have used bilateral LD flaps, conventional and extended, for breast reconstruction, but try to avoid these procedures if possible, although a recent publication suggests that the procedure can be performed without significant impairment of shoulder function [22]. In the last decade, we have increasingly performed bilateral propeller TDAP flaps for breast reconstruction [33]. This procedure requires the patient to be turned twice during surgery.

The evolving TDA flap techniques, starting with the conventional LD flap and ending with the TDAP flap, were driven by the aim of achieving breast reconstructions without including

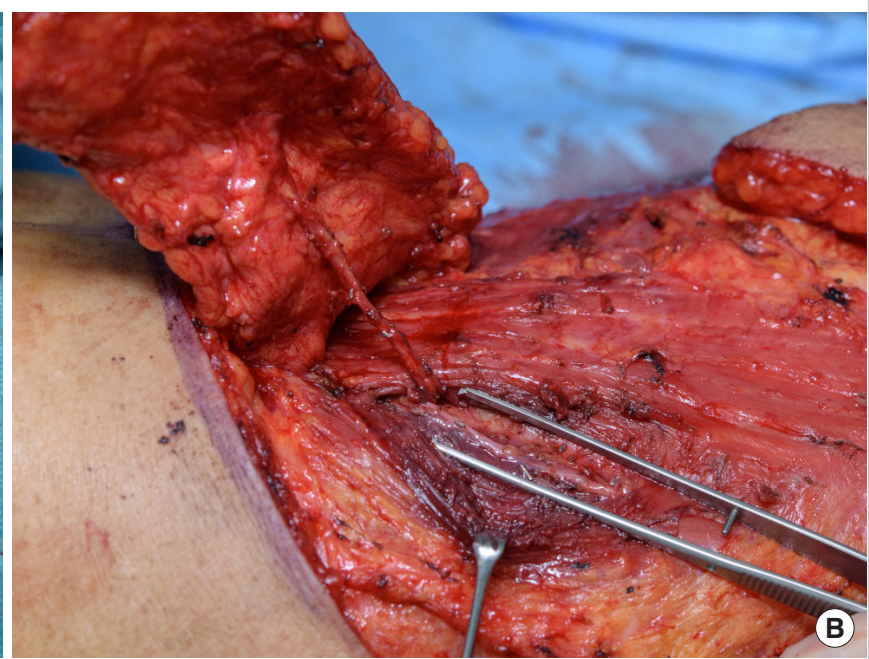

the LD muscle, which is the largest muscle in the upper body [7, $24,26,36]$. For many years we were taught that LD flaps should not be used in physically active women as they require this muscle for sports $[37,38]$. However, this limitation in indication does not make sense, as all women use their LD muscles in everyday life and daily activities. The morbidity associated with harvesting the LD muscle has been examined over time, but the literature relating to this subject is surprisingly divergent. $\mathrm{Re}$ cently, the effect of breast reconstruction with either of these two flaps on shoulder function has been examined $[15,20]$. These studies showed a significant difference in overall shoulder function between the two surgical techniques in favor of the propeller TDAP flap, but surprisingly there was no difference when measuring muscle strength before and after surgery. The finding of LD harvest-associated morbidity is supported by systematic reviews of the literature on LD flaps $[39,40]$. However, the evidence on functional morbidity associated with LD flap harvest is still ambiguous [22,37,40-43]. The follow-up time in most studies examining morbidities and consequences of harvesting the LD flap is relatively short. We need studies with long follow-up to fully understand the consequences of using this flap. The most important question is probably how these women function when they get older, as shoulder function declines with age. There are currently no long-term studies investigating morbidity related to harvest of the LD muscle.

\section{CONCLUSIONS}

The TDA flaps, including the TDAP flap, propeller TDAP flap, the MSLD flap, the LD flap, and the extended LD flap, are all 
important flaps for reconstruction of the breast. The LD flap is still an option, although we prefer flaps without muscle when possible. The vascular-pedicled TDAP flap is an option for experienced surgeons in selected cases for total breast reconstruction and oncoplastic procedures including medial defects. Guided by CDU, the oblique perforator-pedicled or subcutaneous-pedicled propeller TDAP flap can be used in most reconstructive cases of the breast; however, a secondary procedure is often necessary for correction of the pedicle bulk. The extended LD flap is an option for total breast reconstruction without an implant for women with a substantial BMI and substantial fat deposits in the subcutis of the back, although it is associated with the highest morbidity of all the TDA flaps. The MSLD flap can be used if the perforators are small or if dissection of the perforators is assessed to be hazardous.

\section{NOTES}

\section{Conflict of interest}

No potential conflict of interest relevant to this article was reported.

\section{Ethical approval}

The study was approved by the Institutional Review Board of Instituto Oncológico Henry Moore (IRB No. HM-12008) and Danish Committee on Health Research Ethics (IRB No. S20120207) and performed in accordance with the principles of the Declaration of Helsinki. Written informed consent was obtained.

\section{Patient consent}

The patients provided written informed consent for the publication and the use of their images.

\section{Author contribution}

Conceptualization: JB Thomsen, A Rancati, C Angrigiani. Data curation: JB Thomsen, A Rancati, C Angrigiani. Formal analysis: JB Thomsen, A Rancati, C Angrigiani. Methodology: all authors. Project administration: JB Thomsen, C Angrigiani. Visualization: JB Thomsen, M Børsen Rindom. Writing - original draft: all authors. Writing - review \& editing: all authors.

\section{ORCID}

Jørn Bo Thomsen https://orcid.org/0000-0002-7368-6133 Mikkel Børsen Rindom https://orcid.org/0000-0003-3943-8344

Alberto Rancati https://orcid.org/0000-0003-3317-9521

Claudio Angrigiani https://orcid.org/0000-0002-4333-7311.

\section{REFERENCES}

1. Tansini I. Sopra il mio nuovo processo di amputazione della mamella. Riforma Medica 1906;12:757.

2. Maxwell GP. Iginio Tansini and the origin of the latissimus dorsi musculocutaneous flap. Plast Reconstr Surg 1980;65: 686-92.

3. Schneider WJ, Hill HL Jr, Brown RG. Latissimus dorsi myocutaneous flap for breast reconstruction. Br J Plast Surg 1977;30:277-81.

4. Hokin JA. Mastectomy reconstruction without a prosthetic implant. Plast Reconstr Surg 1983;72:810-18.

5. Godina M. The tailored latissimus dorsi free flap. Plast Reconstr Surg 1987;80:304-6.

6. Hayashi A, Maruyama Y. The "reduced" latissimus dorsi musculocutaneous flap. Plast Reconstr Surg 1989;84:290-5.

7. Angrigiani C, Grilli D, Siebert J. Latissimus dorsi musculocutaneous flap without muscle. Plast Reconstr Surg 1995; 96:1608-14.

8. Hamdi M, Van Landuyt K, Monstrey S, et al. Pedicled perforator flaps in breast reconstruction: a new concept. $\mathrm{Br} \mathrm{J}$ Plast Surg 2004;57:531-9.

9. Hamdi M, Salgarello M, Barone-Adesi L, et al. Use of the thoracodorsal artery perforator (TDAP) flap with implant in breast reconstruction. Ann Plast Surg 2008;61:143-6.

10. Gunnarsson GL, Holm J, Duus N, et al. Propeller TAP flap breast reconstruction: a simplified surgical technique. J Plast Reconstr Aesthet Surg 2018;71:1424-31.

11. Jacobs J, Borsen-Koch M, Gunnarsson GL, et al. The versatile extended thoracodorsal artery perforator flap for breast reconstruction. Ann Plast Surg 2016;77:396-400.

12. Borsen-Koch M, Gunnarsson GL, Udesen A, et al. Direct delayed breast reconstruction with TAP flap, implant and acellular dermal matrix (TAPIA). J Plast Reconstr Aesthet Surg 2015;68:815-21.

13. Thomsen JB, Bille C, Wamberg P, et al. Propeller TAP flap: is it usable for breast reconstruction? J Plast Surg Hand Surg 2013;47:379-82.

14. Nahabedian MY, Momen B, Galdino G, et al. Breast reconstruction with the free TRAM or DIEP flap: patient selection, choice of flap, and outcome. Plast Reconstr Surg 2002; 110:466-75.

15. Rindom MB, Gunnarsson GL, Lautrup MD, et al. Shoulderrelated donor site morbidity after delayed breast reconstruction with pedicled flaps from the back: an open label randomized controlled clinical trial. J Plast Reconstr Aesthet Surg 2019;72:1942-9.

16. Angrigiani C, Rancati A, Escudero E, et al. Extended thora- 
codorsal artery perforator flap for breast reconstruction. Gland Surg 2015;4:519-27.

17. Rindom MB, Gunnarsson GL, Lautrup MD, et al. Shoulderrelated donor site morbidity and patient-reported satisfaction after delayed breast reconstruction with pedicled flaps from the back: a comparative analysis. J Plast Reconstr Aesthet Surg 2018;71:1108-15.

18. Angrigiani C, Rancati A, Escudero E, et al. Propeller thoracodorsal artery perforator flap for breast reconstruction. Gland Surg 2014;3:174-80.

19. Ayestaray B, Ogawa R, Ono S, et al. Propeller flaps: classification and clinical applications. Ann Chir Plast Esthet 2011; 56:90-8.

20. Rifaat MA, Amin AA, Bassiouny M, et al. The extended latissimus dorsi flap option in autologous breast reconstruction: a report of 14 cases and review of the literature. Indian J Plast Surg 2008;41:24-33.

21. Chang DW, Youssef A, Cha S, et al. Autologous breast reconstruction with the extended latissimus dorsi flap. Plast Reconstr Surg 2002;110:751-9.

22. Lohana P, Button J, Young D, et al. Functional recovery after bilateral extended autologous latissimus dorsi breast reconstruction: a prospective observational study.J Plast Reconstr Aesthet Surg 2019;72:1060-6.

23. Angrigiani C, Rancati A, Artero G, et al. TDAP: island versus propeller.J Plast Reconstr Aesthet Surg 2016;69:506-11.

24. Thomsen JB, Gunnarsson GL. The evolving breast reconstruction: from latissimus dorsi musculocutaneous flap to a propeller thoracodorsal fasciocutaneous flap. Gland Surg 2014;3:151-4.

25. Gunnarsson GL, Borsen-Koch M, Nielsen HT, et al. Bilateral breast reconstruction with extended thoracodorsal artery perforator propeller flaps and implants. Plast Reconstr Surg Glob Open 2015;3:e435.

26. Perignon D, Qassemyar Q Benhaim T, et al. From Tansini to Angrigiani: improvement and refinement of the thoracodorsal flap. Ann Chir Plast Esthet 2011;56:149-55.

27. Angrigiani C, Rancati A, Artero G, et al. Stacked thoracodorsal artery perforator flaps for unilateral breast reconstruction. Plast Reconstr Surg 2016;138:969e-972e.

28. Hamdi M. Oncoplastic and reconstructive surgery of the breast. Breast 2013;22 Suppl 2:S100-5.

29. Adler N, Seitz IA, Song DH. Pedicled thoracodorsal artery perforator flap in breast reconstruction: clinical experience. Eplasty 2009;9:e24.

30. Ortiz CL, Mendoza MM, Sempere LN, et al. Versatility of the pedicled thoracodorsal artery perforator (TDAP) flap in soft tissue reconstruction. Ann Plast Surg 2007;58:315-20.
31. Borsen-Koch M, Gunnarsson GL, Sorensen JA, et al. Thermal injury in TAPIA breast reconstruction-thermal injury to thoracodorsal artery perforator flap. Gland Surg 2017;6:110-3.

32. Gunnarsson GL, Tei T, Thomsen JB. Color doppler ultrasonography-targeted perforator mapping and angiosomebased flap reconstruction. Ann Plast Surg 2016;77:464-8.

33. Lorenzen MM, Gunnarsson GL, Bille C, et al. Visualized bilateral breast reconstruction by propeller thoracodorsal artery perforator flaps. Gland Surg 2019;8(Suppl 4):S262-70.

34. Ibrahim RM, Gunnarsson GL, Akram J, et al. Color Doppler ultrasonography targeted reconstruction using pedicled perforator flaps: a systematic review and meta-analysis. Eur J Plast Surg 2018;41:495-504.

35. Alstrup T, Christensen BO, Damsgaard TE. ICG angiography in immediate and delayed autologous breast reconstructions: peroperative evaluation and postoperative outcomes. J Plast Surg Hand Surg 2018;52:307-11.

36. Abdelrahman EM, Nawar AM, Balbaa MA, et al. Oncoplastic volume replacement for breast cancer: latissimus dorsi flap versus thoracodorsal artery perforator flap. Plast Reconstr Surg Glob Open 2019;7:e2476.

37. Lutringer J, Flipo B, Carles M, et al. Functional impairment and its impact on sporting activities after latissimus dorsi flap breast reconstruction. Ann Chir Plast Esthet 2012;57: 567-74.

38. Garusi C, Manconi A, Lanni G, et al. Shoulder function after breast reconstruction with the latissimus dorsi flap: a prospective cohort study. Combining DASH score and objective evaluation. Breast 2016;27:78-86.

39. Blackburn NE, Mc Veigh JG, Mc Caughan E, et al. The musculoskeletal consequences of breast reconstruction using the latissimus dorsi muscle for women following mastectomy for breast cancer: a critical review. Eur J Cancer Care (Engl) 2018;27:e12664.

40. Smith SL. Functional morbidity following latissimus dorsi flap breast reconstruction. J Adv Pract Oncol 2014;5:181-7.

41. Friedman O, Fliss E, Inbal A, et al. Latissimus dorsi flap: a winning hand for breast reconstruction salvage. Isr Med Assoc J 2019;21:260-4.

42. Umar M, Jahangir N, Hughes M, et al. Incidence of shoulder functional morbidity following ipsilateral mastectomy and latissimus dorsi flap reconstruction. Acta Orthop Traumatol Turc 2019;53:448-51.

43. Browne JP, Jeevan R, Pusic AL, et al. Measuring the patient perspective on latissimus dorsi donor site outcomes following breast reconstruction. J Plast Reconstr Aesthet Surg 2018;71:336-43. 